\title{
Plasmons of Hexamer and Pentamer Nanocavities Probed with Swift Electrons
}

\author{
Nahid Talebi ${ }^{1}$, Burcu Ögüt ${ }^{1}$, Wilfried Sigle ${ }^{1}$, Ralf Vogelgesang ${ }^{2}$, and Peter A. van Aken ${ }^{1}$ \\ 1. Max Planck Institute for Intelligent Systems, Stuttgart, Germany \\ 2. University of Oldenburg, Oldenburg, Germany
}

Electron energy-loss spectroscopy (EELS) is an efficient tool for investigating the local density of optical states in single and coupled nano-systems in a transmission electron microscope (TEM) [1]. In EELS, a relativistic electron inelastically interacts with a sample, and hence loses energy by pumping the sample to a higher photonic state. The amount of energy loss of the electron is detected, providing us with information about the resonant energies of the sample. Mapping EELS by parallel acquisition, energy-filtered transmission electron microscopy (EFTEM) is a fast and efficient detection tool for mapping the optical modes in two spatial dimensions. Here, this is made possible by the in-column MANDOLINE energy filter in the Zeiss SESAM microscope [2].

Using EFTEM, we have analyzed the plasmon modes of hexamer and pentamer nanocavities as shown in Figure 1, especially with respect to their symmetry and topology rules. The pentamer nanocavity is composed of holes with diameters of $60 \mathrm{~nm}$ and rim-to-rim spacing of $55 \mathrm{~nm}$ drilled into a silver slab of $100 \mathrm{~nm}$ thickness. Selected images of the acquired EFTEM series are depicted in Figure 1 (a). By utilizing a peak-finding algorithm [3], the spatial distribution of the excited modes can be efficiently investigated. It is clear that four distinguished plasmon resonances at the mapped energy are excited, which is due to the modes of various symmetries as determined by the electric dipoles oriented along the radial or azimuthal directions. In order to more precisely analyse the plasmon eigenenergies, the reported EELS spectra at certain electron impacts are compared to theoretically computed EELS spectra, utilizing a FDTD method with an embedded electron source [4], as shown in Figure 1 (c). The bulk plasmon exhibits a broad resonance centred at $3.5 \mathrm{eV}$ for silver. As it is visible from the peak maps, the radially polarized plasmon mode has a resonant energy of $3.4 \mathrm{eV}$. The interference of these two modes becomes visible in the calculated EELS spectra by a typical Fano-shaped resonance.

Figure 1 (d) shows the EFTEM series for an oligomer nanocavity composed of 6 holes drilled in a 100 $\mathrm{nm}$ silver slab. The diameters of the holes are $70 \mathrm{~nm}$, and the rim-to-rim spacing between the holes is 30 nm. A comparison between the peak maps depicted in Figure 1 (e) with those of the heptamer nanocavity shows that again the same classification of the modes into longitudinally and radially polarised modes is possible. There is no evidence of a toroidal mode as reported in ref. [5] for an oligomer nanocavity with a central hole, which is a proof of a strong dependence of this mode on the topology of the structure. The experimental and calculated EELS spectra show a blue shift of the resonances with respect to the pentamer nanocavity, which is a specification of the shorter spacing, and hence a stronger coupling of the nanoholes.

Our investigations have shown that there is no proof of an electron-driven optical mode which can be related to the 6-fold symmetry of a hexamer, in comparison with the 5-hole symmetry of the pentamer. This fact is mainly due to the short circumference of the plasmonic rings of holes, in such a way that the interference of the rotating plasmons along the circumference as shown in [6] is not feasible [7]. 


\section{References:}

[1] J Garcia de Abajo, Rev. Mod. Phys. 82 (2010), p. 209.

[2] S Uhlemann and H Rose, Ultramicroscopy 63 (1996) p. 161.

[3] N Talebi, et al, Langmuir 28 (2012), p. 8867.

[4] N Talebi, et al, New J. Phys. 15 (2013) 053013.

[5] B Ögüt, et al, Nano Lett. 12 (2012) p. 5239.

[6] N Talebi, A Mahjoubfar, and M. Shahabadi, J. Opt. Soc. Am. B 25 (2008) p. 2116.

[7] NT acknowledges the Alexander-von-Humboldt Foundation for financial support. The research leading to these results has received funding from the European Union Seventh Framework Programme [FP7/2007-2013] under grant agreement n³12483 (ESTEEM2).

(a)

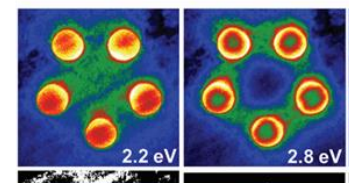

(b)
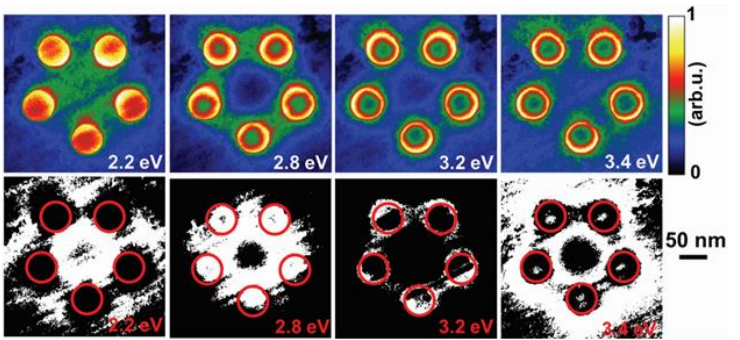

(c)
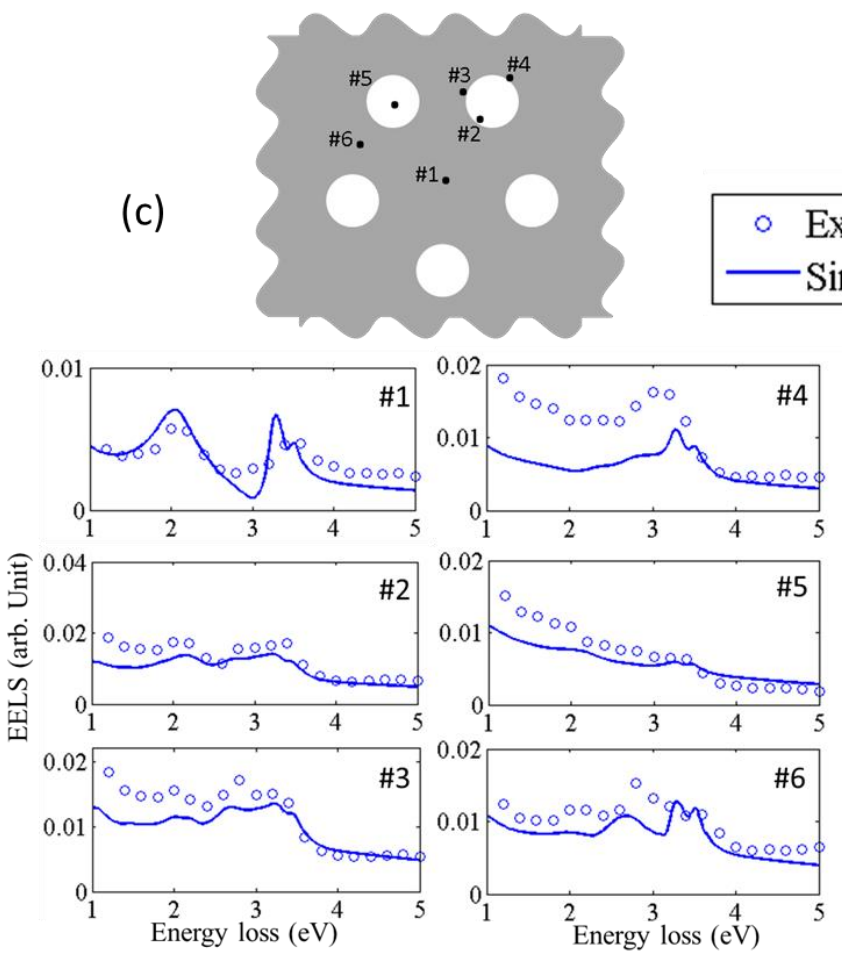

(d)

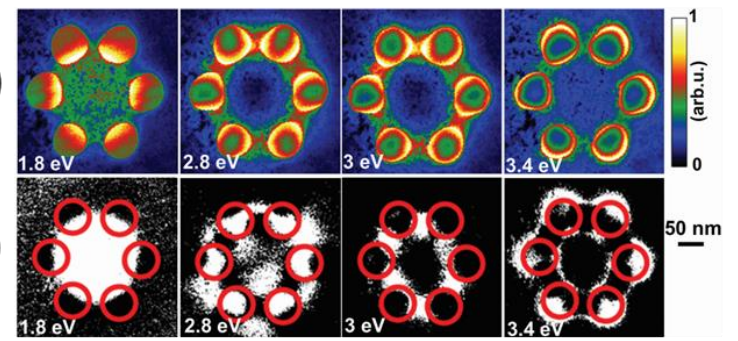

(f)
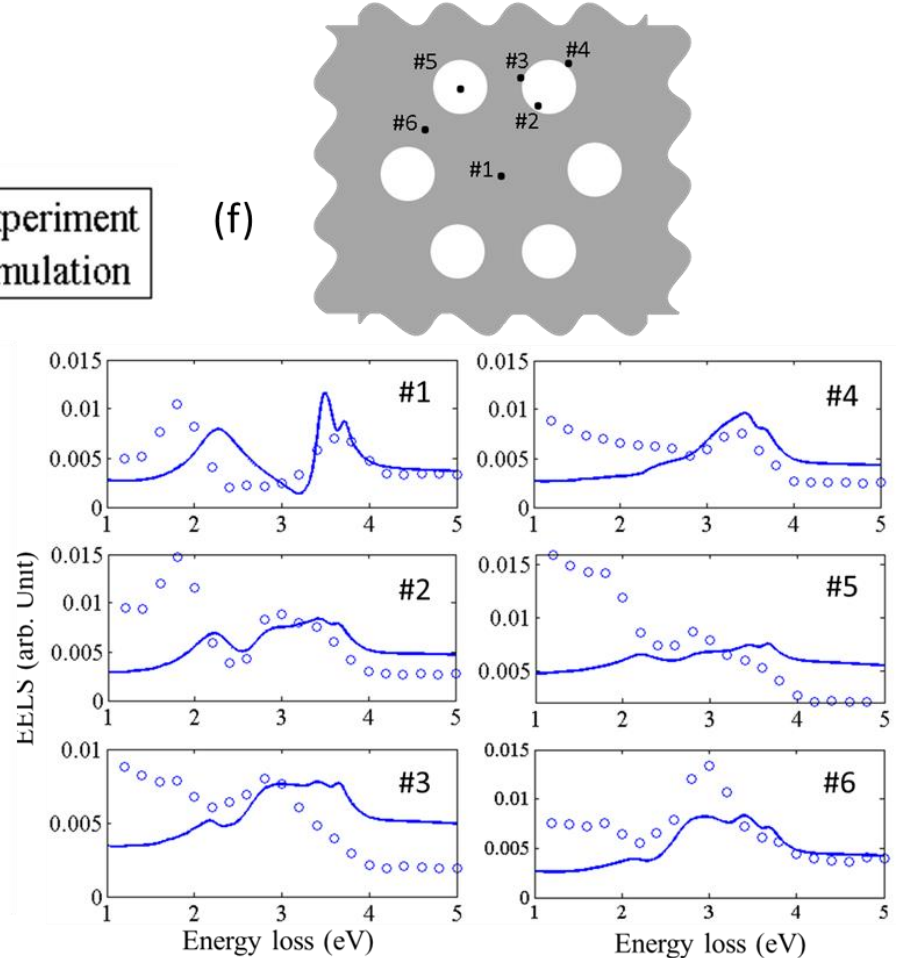

Figure 1. (a) EFTEM series at depicted energies, (b) peak-maps for the acquired EFTEM series, and (c) experimental and calculated EELS spectra acquired for a void pentamer nanocavity composed of nanoholes with a diameter of $60 \mathrm{~nm}$ and rim-to-rim spacing of $55 \mathrm{~nm}$, drilled into a silver slab of 100 nm thickness. (d) EFTEM series at depicted energies, (b) peak maps for the acquired EFTEM series, and (c) experimental and calculated EELS spectra acquired for a void hexamer nanocavity composed of nanoholes with a diameter of $70 \mathrm{~nm}$ and rim-to-rim spacing of $30 \mathrm{~nm}$, drilled into a silver slab of 100 nm thickness. 